\title{
Subjective sleep quality, quantitative sleep features, and their associations dependent on demographic characteristics, habitual sleep-wake patterns, and distinction of weekdays/weekends
}

\author{
Siwalee Choilek ${ }^{1} \cdot$ Akihiro Karashima $^{2} \cdot$ Ikuko Motoike $^{1,3} \cdot$ Norihiro Katayama $^{1} \cdot$ Kengo Kinoshita $^{1,3}$. \\ Mitsuyuki Nakao ${ }^{1}$ i
}

Received: 16 July 2020 / Accepted: 23 April 2021 / Published online: 9 May 2021

(c) The Author(s) 2021

\begin{abstract}
Generally applicable qualifications of sleep and principles for achieving better sleep are difficult to design, because sleep quality can depend on individual demographic characteristics and lifestyles. In this study, the static and dynamic features of sleep-wake patterns were analyzed in association with quantitative sleep-related parameters and self-rated sleep quality to serve as a practical selection of sleep-wake patterns fitted to individual conditions. Data obtained over a 2-week period by actigraphy from university students and information technology workers were measured to obtain a daily subjective rating of sleep quality using the Oguri-Shirakawa-Azumi (OSA) sleep inventory. Qualitative sleep quality in terms of OSA score and quantitative sleep-related and chronobiological features were analyzed with regard to their dependency on the demographic characteristics, habitual sleep-wake patterns (HSWP), and distinction of weekdays/weekends. Multi-factor ANOVA was used to further investigate their dependencies regarding multiple ways of interactions between the demographic characteristics, HSWP, and distinction of weekdays/weekends. Subjective sleep quality and quantitative sleep-related parameters depended on the demographic characteristics, and so did their associations. The classification of day-to-day variations in HSWP showed four clusters that were effective factors for understanding their dependencies. Multi-factor analysis revealed demographic characteristics, HSWP, distinction of weekdays/weekends, and their multi-way interactions up to 3rd order as significant effectors of qualitative and quantitative quality of sleep. This study clarified how quantitative sleep-related parameters, subjective sleep quality, and their associations depended on demographic characteristics. Furthermore, their dependency was understood as a combination of multi-way interactions between the demographic characteristics, HSWP, and the distinction of weekdays/weekends. Our findings could provide a basis for the design of individually matched sleep-wake patterns.
\end{abstract}

Keywords Actigraphy $\cdot$ OSA $\cdot$ Clustering of sleep-wake patterns $\cdot$ Multi-way interactions

\section{Introduction}

Sleep plays an important role in the maintenance of human mental and physical conditions. Poor sleep quality can cause wide range health problems, both acute (e.g., mood swings, slower cognitive response, higher stress levels) and chronic (e.g., obesity, increased risk of cardiometabolic diseases) [1-3]. How to achieve good sleep quality has been attracting increasing attention, and numerous theories and methods have been proposed [4]. Among these, wearable devices can inform users of their estimated sleep quality based on daily activity and/or physiological signals such as heart rate, blood pressure, and body temperature [5, 6]. However, people used to select their sleep-wake patterns suitable for individual conditions according to one's own empirical ideas. Of 
course, good sleep should take place with appropriate timing and length in a proper environment, with no disruptions [7]. However, because demographic characteristics, working style, individual preferences, and physiological responsiveness in terms of sleep regulation vary from person to person, generally applicable sleep qualifications and principles for achieving better sleep are difficult to design.

Chronotype and social jet lag (SJL) have recently become widely recognized as chronobiological parameters that characterize habitual sleep-wake patterns [2]. People with the early chronotype prefer earlier sleep phases, and vice versa [8-11]. Naturally, those with the late chronotype suffer from insufficient sleep, and show a tendency to accumulate sleep debt over working days that results in prolonged sleep on the weekend or non-working days, i.e., severe SJL [11]. Several studies have suggested that the late chronotype and severe SJL influence physiological phenomena such as later melatonin secretion [9], later circadian acrophase of activity [10], and lower sleep regularity $[12,13]$, which could underlie the deterioration of sleep quality. Therefore, these parameters may be possible candidates of factors that relate habitual sleep-wake patterns to the quality of sleep. As sleep is dynamically regulated by interactions between circadian rhythms and homeostasis under the influence of environmental and social time cues [14], it might be useful to employ more dynamical features of habitual sleep-wake patterns, rather than static parameters such as chronotype and SJL, in designing sleep-wake patterns for maintaining/improving sleep quality. In fact, the dynamical feature of regularity in the daily sequence of resting states has been suggested as a relevant index in the evaluation of health conditions [3,15].

We analyzed the association of quantitative feature parameters of activity during sleep, static chronobiological parameters such as chronotype and SJL, and self-rated sleep quality with demographic characteristics and day-today variations in habitual sleep-wake patterns. The identified associations were dissected into multiple interactions between these parameters and factors. The present results could contribute to the practical selection of sleep-wake patterns fitted to individual conditions, including demographic characteristics, individual preferences, working style, and chronobiological/homeostatic regulation.

\section{Materials and methods}

The data were acquired in 2014-2017, partly from a previous study conducted under a different context [16]. The 356 participants with no severe sleep complaints were recruited in the study, including information technology workers $(N=180)$ or university students $(N=176)$ aged $20-63$ years. All participants were asked to record their physical activity (sampling rate: $30 \mathrm{~Hz}$ ) using a wrist-mounted accelerometer (Actigraph ${ }^{\circledR}$ GT3X + ; Actigraph LLC, FL, USA) and complete the Oguri-Shirakawa-Azumi (OSA) sleep questionnaire [17] upon waking, for 15 days. The OSA sleep questionnaire quantifies subjective sleep quality into five categories: sleepiness at awakening (OSA-1), sleep maintenance (OSA-2), dreaming (OSA-3), recovery from fatigue (OSA-4), and self-reported sleep duration (OSA-5). Higher OSA scores reflect better sleep quality. All measurement procedures were conducted under the approval of the Ethical Committee of the Graduate School of Information Sciences, Tohoku University, Japan.

\section{Estimation of quantitative sleep-related parameters}

Sleep-wake timing and parameters were estimated using ActiLife ${ }^{\circledR}$ (Actigraph LLC, FL, USA) based on the Cole-Kripke sleep-wake identification algorithm [18]. A sleep report using the sensor algorithm comprised wake after sleep onset (WASO), sleep-onset latency (SOL), number of awakenings (NAWK), and sleep efficiency (SE) [19]. Midsleep time (MS), chronotype ( $\mathrm{MSF}_{\mathrm{sc}}$; mid-sleep on free days corrected for sleep debt on workdays), and SJL were also calculated as described in $[2,8,20]$. A simplified version of the Sleep Regularity Index (SRI) was used to estimate a mean coincidence percentage of sleep periods between 2 consecutive days [12].

To extract the onset of posture change during sleep, the data were multiband-filtered (finite impulse response [FIR] filter of order 20; pass band, $0-0.05$ and $2-5 \mathrm{~Hz}$ ) and then segmented into 2 -s bins with $50 \%$ overlap. The mean and variance of the filtered signals were calculated for each bin. Movement in each bin at time $t$ was detected if the statistical quantities for at least one of three-dimensional signals satisfied either of the following conditions: (1) the absolute difference between the successive mean levels at $t$ and $t+1$ was larger than the threshold $(=0.01)$, or $(2)$ the variance at $t$ was larger than the threshold $(=0.007)$. Movements lasting for less than $4 \mathrm{~s}$ and intermissions of less than $3 \mathrm{~s}$ were ignored, thus excluding jitter-like movements and subjecting only major posture changes for analysis. Figure S-1a in Online Resource 1 shows the method used to estimate inter-movement-onset intervals (IMOIs). IMOIs were subjected to logarithmic transformation because of their longtailed distribution, and then, their mean, $\mathrm{T}_{\text {mean }}$, and standard deviation (SD), $\mathrm{T}_{\text {std }}$, were calculated for each sleep episode. In addition, the number of movements, $\mathrm{N}_{\mathrm{m}}$, and movement density, $\mathrm{D}_{\mathrm{m}}$ (ratio of number of movements to sleep length), were obtained for each sleep episode.

The circadian amplitude and acrophase of daily activity were estimated based on one-component static cosine fitting with 24-h period. Daily activity sequences were band-pass filtered (FIR filter of order 30; pass band: $2-5 \mathrm{~Hz}$ ), and the locomotor inactivity during sleep (LIDS) ultradian rhythm 
was then extracted following a previously reported method [21]. The parameters obtained were ultradian period, LIDS slope, range of oscillation (RoO), and number of ultradian cycles $\left(\mathrm{N}_{\mathrm{c}}\right)$. Figure $\mathrm{S}-1 \mathrm{~b}$ shows an example of an ultradian rhythm fitted to the LIDS.

\section{Statistical analysis and classification of habitual sleep-wake patterns (HSWPs)}

All statistical analyses were performed using MATLAB (R2017a). The activities and OSA scores were analyzed for 169 participants (44 male students [age: 21.9 (mean) \pm 1.60 (SD)], 38 female students [21.4 \pm 1.45$]$, 66 male workers $[44.3 \pm 10.4], 21$ female workers [39.5 \pm 11.1$]$ ) in whom these data were recorded for 7 consecutive days. An omnibus test for the demographics dependency of subjective sleep quality and quantitative sleep-related features was performed using one-way analysis of variance (ANOVA) or the Kruskal-Wallis test depending on the distribution of the parameters. This was followed by $t$ test or Mann-Whitney $U$ test, respectively, as post hoc tests (Table 1 and Table S-1 of Online Resource 2). Following multiple comparisons as host hoc analysis, the Bonferroni correction was used (Tables S-1 and S-3).

The associations between quantitative sleep-related features and OSA scores were analyzed for 305 participants (95 male students [age: 21.9 (mean) \pm 1.83 (SD)], 55 female students [21.2 \pm 1.42 ], 116 male workers [41.0 \pm 11.0$], 39$ female workers $[38.5 \pm 10.0]$ ) in whom activities and OSA scores were recorded for $\geq 10$ days (Tables $2,3,4,5$, and Table S-2 of Online Resource 2), where the female workers were not subjected to analysis for stratification reasons. Individual OSA scores were standardized (z-score) before estimating the Spearman correlations separately for gender distinction in the students and age group distinction in the male workers. The male workers were grouped by age into three groups $(20 \leq$ age $<35,35 \leq$ age $<45$, and $45 \leq$ age $\leq 63)$, considering the age distribution of workers in which there was a gap around 35 (Fig.S-3 of Online Resource 2). This grouping did not so deviate from commonly used every $\sim 10$ years grouping [22]. The same age grouping was applied in another analysis (Table S-1 of Online Resource 2).

Of the 356 participants, HSWPs were classified in 290 (83 male students [age:22.0 (mean) \pm 2.14 (SD)], 60 female students [21.3 \pm 1.45$], 107$ male workers [42.6 \pm 10.5$], 40$ female workers $[38.5 \pm 10.7])$ who had completed $\geq 14$ consecutive days of activity recordings, out of which 14 consecutive days were subjected to the following clustering. $\mathrm{K}$-means clustering was then performed using 10-dimensional features, including the mean and SD of sleep- and wake-onset times, second and third harmonics of the sleepand wake-onset time daily sequences, average MS, and $\mathrm{MSF}_{\mathrm{sc}}$. Then, principal component analysis (PCA) was applied to show the clustering result explicitly in a lower dimensional space. Among the variables used for clustering, the former 8 variables were selected as those for effectively characterizing HSWP. The latter two variables were selected out of chronotype (MSFsc), variation of mid-sleep time, SRI, and variation of sleep duration, because including these two variables yielded the best result in terms of percentage of variance explained in PCA. Appropriate numbers of clusters and cluster features were confirmed by hierarchical clustering and the elbow method [23].

To summarize the characterization of subjective sleep quality and quantitative sleep-related features, their multiple dependency on demographic characteristics and HSWP as between-subject factors, and distinction of weekdays/ weekends as a within-subject factor were investigated by mixed factor ANOVA [24], where the data of individual subjects were averaged separately for weekdays or weekends, and a repeat measure model was then generated. The model included both main and interaction effects among factors. In addition, the post hoc analysis was done by Tukey-Kramer test.

\section{Results}

\section{Demographics dependency of quantitative sleep-related parameters and subjective sleep quality, and their associations}

Among the sleep-related parameters, the distributions of the overnight average of IMOIs and ultradian rhythm parameters of LIDS, including the ultradian period, RoO, and slope, are shown in Fig. S-2 of Online Resource 1. Comparisons across the four different subject groups are listed in Table 1. Characteristically, demographics dependency of quantitative sleep-related parameters was more pronounced than that of subjective sleep quality. Distinctively significant difference in subjective sleep quality was confined to dreaming frequency (OSA-3) between male students and workers, and sleepiness at awakening between female students and workers. Irrespective of gender, students tended to take a later, longer, more irregular, and less disturbed sleep than the workers. Gender difference was observed in a job-specific way, i.e., male students tended to take a later and more disturbed sleep than female students. In contrast, the sleep of male workers tended to suffer more dense disturbances than that of female workers. Because the ages of male workers were distributed over a wide range, they were separated into three generational groups and age dependency was analyzed (Table S-1 of Online Resource 2). Sleep timings tended to be earlier in the eldest group than in other groups, which was also suggested by the chronobiological features. The flat ultradian slope also characterized the sleep of the eldest 
Table 1 Comparisons with regard to gender difference (male/female) and job difference (students/workers)

\begin{tabular}{|c|c|c|c|c|c|c|c|c|}
\hline Parameters & $\begin{array}{l}\text { Male students } \\
(44)\end{array}$ & $\begin{array}{l}\text { Female students } \\
(38)\end{array}$ & $\begin{array}{l}\text { Male workers } \\
(66)\end{array}$ & $\begin{array}{l}\text { Female workers } \\
\text { (21) }\end{array}$ & $\begin{array}{l}\text { Gender comp. } \\
\text { (students) }\end{array}$ & $\begin{array}{l}\text { Gender comp } \\
\text { (workers) }\end{array}$ & Job comp (male) & Job comp (female) \\
\hline OSA-1 & $15.80[3.61]$ & $15.25[4.72]$ & $17.75[3.75]$ & $15.80[3.50]$ & $\mathrm{Z}=2.49 *$ & $Z=-0.05$ & $Z=-1.73$ & $\mathrm{Z}=-3.3 * *$ \\
\hline OSA-2 & $18.20[2.50]$ & $17.60[3.30]$ & $18.40[3.30]$ & $17.60[2.90]$ & $\mathrm{Z}=1.79$ & $Z=2.06^{*}$ & $Z=-1.51$ & $Z=-0.8$ \\
\hline OSA-3 & $23.50[5.00]$ & $23.50[5.00]$ & $29.50[3.00]$ & $23.50[5.00]$ & $Z=0.45$ & $\mathrm{Z}=1.72$ & $\mathrm{Z}=-3.03 * *$ & $Z=-0.9$ \\
\hline OSA-4 & $15.67[3.35]$ & $15.67[4.83]$ & $15.70[4.85]$ & $15.70[4.85]$ & $\mathrm{Z}=0.21$ & $Z=-0.38$ & $Z=-1.85$ & $Z=-1.9$ \\
\hline OSA-5 & $16.00[5.50]$ & $16.00[5.50]$ & $16.00[5.50]$ & $16.00[5.50]$ & $Z=-0.23$ & $\mathrm{Z}=0.92$ & $Z=-1.6$ & $\mathrm{Z}=-0.1$ \\
\hline $\begin{array}{l}\text { Sleep onset } \\
\quad(\mathrm{hh}: \mathrm{mm})\end{array}$ & $01: 36(01: 32)$ & $00: 56[01: 07]$ & $00: 21(01: 29)$ & $00: 22(01: 15)$ & $\mathrm{Z}=3.71 * * *$ & $\mathrm{t}(607)=-0.09$ & $\mathrm{t}(768)=11.24 * * *$ & $\mathrm{Z}=4.4 * * *$ \\
\hline $\begin{array}{l}\text { Wake onset } \\
\text { (hh:mm) }\end{array}$ & $08: 04[00: 54]$ & $07: 59[01: 00]$ & $06: 33[00: 42]$ & $06: 27[00: 33]$ & $\mathrm{Z}=2.04 * *$ & $Z=1.62$ & $\mathrm{Z}=14.27 * * *$ & $\mathrm{Z}=9.56^{* * *}$ \\
\hline $\begin{array}{l}\text { Sleep } \\
\text { duration } \\
\text { (hh:mm) }\end{array}$ & $06: 43(01: 24)$ & $06: 57(01: 39)$ & $06: 26[00: 52]$ & $06: 16(01: 26)$ & $\mathrm{t}(572)=-1.81$ & $\mathrm{Z}=1.19$ & $\mathrm{Z}=3.11^{* *}$ & $\mathrm{t}(411)=4.2 * * *$ \\
\hline MS (hh:mm) & $04: 50[00: 51]$ & $04: 30[00: 53]$ & $03: 28[00: 46]$ & $03: 20[00: 29]$ & $\mathrm{Z}=3.47 * * *$ & $Z=0.62$ & $\mathrm{Z}=13.64^{* * *}$ & $\mathrm{Z}=8.05^{* * *}$ \\
\hline $\begin{array}{l}\mathrm{MSF}_{\mathrm{SC}} \\
\quad(\mathrm{hh}: \mathrm{mm})\end{array}$ & 05:05(01:19) & $04: 46(01: 26)$ & $03: 46(01: 29)$ & 03:55(01:05) & $t(80)=1$ & $t(85)=-0.42$ & $\mathrm{t}(108)=4.69 * * *$ & $t(57)=2.33^{*}$ \\
\hline SJL (hh:mm) & $00: 26[00: 30]$ & $00: 44(00: 38)$ & 01:08(00:52) & 01:01(00:45) & $Z=-0.14$ & $\mathrm{t}(85)=0.47$ & $Z=-2.63^{* *}$ & $t(57)=-1.5$ \\
\hline SRI (\%) & $76.28[8.12]$ & $78.17[7.83]$ & $79.04[5.37]$ & $81.65[5.05]$ & $Z=-0.39$ & $Z=-1.58$ & $\mathrm{Z}=-1.51$ & $Z=-1.8$ \\
\hline SE $(\%)$ & $92.83[3.16]$ & $94.77[2.85]$ & $91.28[4.26]$ & $91.05[4.36]$ & $\mathrm{Z}=-5.93^{* * *}$ & $Z=-0.18$ & $\mathrm{Z}=3.25 * *$ & $\mathrm{Z}=6.31 * * *$ \\
\hline $\begin{array}{l}\text { SOL (min- } \\
\text { utes) }\end{array}$ & $1.00[1.50]$ & $2.00[2.00]$ & $2.00[1.50]$ & $3.00[1.50]$ & $Z=-1.45$ & $Z=0.01$ & $\mathrm{Z}=-6.08 * * *$ & $\mathrm{Z}=-2.8 * *$ \\
\hline $\begin{array}{l}\text { NAWK } \\
\text { (times) }\end{array}$ & $14.50[5.25]$ & $11.00[5.50]$ & $16.00[6.50]$ & $15.00[6.00]$ & $\mathrm{Z}=5.35 * * *$ & $Z=0.39$ & $Z=-0.66$ & $\mathrm{Z}=-4.3^{* * *} *$ \\
\hline $\begin{array}{l}\text { WASO } \\
\quad \text { (minutes) }\end{array}$ & $27.00[13.25]$ & $19.00[11.00]$ & $31.50[18.00]$ & $30.00[17.00]$ & $\mathrm{Z}=5.39 * * *$ & $Z=0.74$ & $Z=-2.02 *$ & $\mathrm{Z}=-4.8^{* * *}$ \\
\hline $\begin{array}{l}\mathrm{T}_{\text {mean }}(\mathrm{sec}- \\
\text { onds) }\end{array}$ & $267.33[53.53]$ & $295.04[55.40]$ & $264.87[64.74]$ & $291.37[73.58]$ & $Z=-2.34$ & $\mathrm{Z}=-3.3^{* *}$ & $\mathrm{Z}=1.32$ & $Z=-0.9$ \\
\hline $\begin{array}{c}\mathrm{T}_{\text {std }}(\mathrm{sec}- \\
\text { onds) }\end{array}$ & $312.35[54.20]$ & $338.73[61.44]$ & $294.40[72.10]$ & $321.53[71.98]$ & $\mathrm{Z}=-3^{* *}$ & $\mathrm{Z}=-2.84 * * *$ & $Z=2.46^{*}$ & $\mathrm{Z}=1.03$ \\
\hline $\mathrm{N}_{\mathrm{m}}$ (times) & $40.00[12.50]$ & $39.00[10.50]$ & $38.00[12.50]$ & $30.00[10.88]$ & $\mathrm{Z}=0.7$ & $\mathrm{Z}=3.86^{* * *}$ & $Z=0.69$ & $\mathrm{Z}=4.01 * * *$ \\
\hline $\mathrm{D}_{\mathrm{m}}$ & $0.10[0.02]$ & $0.09[0.02]$ & $0.10[0.03]$ & $0.09[0.02]$ & $Z=2.28 *$ & $\mathrm{Z}=3.82 * * *$ & $Z=-0.48$ & $\mathrm{Z}=2.14^{*}$ \\
\hline $\begin{array}{l}\text { Ultradian } \\
\text { period } \\
\text { (min) }\end{array}$ & $80.00[15.00]$ & $80.00[12.50]$ & $80.00[12.50]$ & $75.00[15.00]$ & $Z=-0.24$ & $Z=1.64$ & $\mathrm{Z}=0.81$ & $\mathrm{Z}=2.31^{*}$ \\
\hline LIDS slope & $-0.13[0.09]$ & $-0.11[0.09]$ & $-0.11[0.10]$ & $-0.09[0.10]$ & $Z=-0.01$ & $Z=-0.76$ & $Z=-2.03 *$ & $\mathrm{Z}=-2.2^{*}$ \\
\hline $\mathrm{RoO}$ & $7.59[1.77]$ & $6.88[1.62]$ & $7.09[1.62]$ & $6.84[1.57]$ & $\mathrm{Z}=2.72 * *$ & $Z=1.57$ & $Z=2.45^{*}$ & $\mathrm{Z}=0.75$ \\
\hline $\mathrm{N}_{\mathrm{c}}$ & $4.80[1.04]$ & $5.20[0.95]$ & $4.8[1.05]$ & $4.78[1.19]$ & $\mathrm{Z}=-1.27$ & $Z=-0.34$ & $\mathrm{Z}=1.20$ & $Z=1.34$ \\
\hline $\begin{array}{l}\text { Circadian } \\
\text { amplitude }\end{array}$ & $34.15[12.94]$ & $32.99[9.53]$ & $24.15[9.99]$ & $23.61[9.32]$ & $\mathrm{Z}=1.79$ & $Z=-0.6$ & $\mathrm{Z}=8.29 * * *$ & $\mathrm{Z}=4.92 * * *$ \\
\hline $\begin{array}{r}\text { Acrophase } \\
(\mathrm{hh}: \mathrm{mm})\end{array}$ & $16: 00[01: 30]$ & $15: 20[01: 20]$ & $14: 30[01: 30]$ & $14: 00[01: 20]$ & $\mathrm{Z}=3.12 * * *$ & $\mathrm{Z}=1.46$ & $\mathrm{Z}=7.7 * * *$ & $\mathrm{Z}=4.71 * * *$ \\
\hline
\end{tabular}

Values are represented as mean (SD, Gaussian)/median [semi- interquartile range, non-Gaussian]. As post hoc tests, $t$ test, and Mann-Whitney $U$ (MWU) tests were used for Gaussian and non-Gaussian cases, respectively. $t$-value and degree of freedom, and Z-value are given for $t$ test and MWU test, respectively

$* * * p<0.001, * * p<0.01, * p<0.05$. MS: mid-sleep time, $M S F_{\mathrm{SC}}$ mid-sleep on free days corrected for sleep debt on workdays, $S J L$ social jet lag, $S R I$ sleep regulatory index, $S E$ sleep efficiency, $S O L$ sleep-onset latency, $N A W K$ number of awakenings, WASO wake after sleep onset, $N_{\mathrm{m}}$ number of movements, $D_{\mathrm{m}}$ movement density, $L I D S$ locomotor inactivity during sleep, $R o O$ range of oscillation in LIDS, $N_{\mathrm{c}}$ number of cycles in LIDS. Circadian amplitude and Acrophase are estimated by cosine fitting to the daily activity. These abbreviations and notations are applicable for the following tables

group. The sleep of the middle-aged group tended to be less disturbed in terms of NAWK and $\mathrm{N}_{\mathrm{m}}$, accompanying subjective sleep ratings of highest OSA-2 and OSA-3, and lowest OSA-1 and OSA-4 in comparison with the other groups. The youngest group tended to take a longer and later sleep, which coincided with later chronobiological features, although not significantly. However, sleep in this group was characterized quantitatively by frequent disturbances in terms of NAWK and $\mathrm{N}_{\mathrm{m}}$, accompanying subjective sleep ratings of lower OSA-2, 3, and 4.

In correlation analyses performed between daily quantitative sleep-related parameters and subjective sleep quality for each demographic group (Tables 2, 3, 4, 5) (Spearman correlations), the student analysis focused on gender difference 
Table 2 Spearman correlation (male students) between daily quantitative sleep-related parameters and subjective sleep quality
Table 3 Spearman correlation (female students) between daily quantitative sleep-related parameters and subjective sleep quality

\begin{tabular}{llllllc}
\hline SSQ/OSP & $\begin{array}{l}\text { Mean (SD) or } \\
\text { median [semi-iqr] }\end{array}$ & OSA-1 & OSA-2 & OSA-3 & OSA-4 & OSA-5 \\
\hline SE (\%) & $91.84[3.36]$ & -0.002 & $0.115^{* * * *}$ & 0.027 & 0.001 & 0.04 \\
SOL (min) & $2.00[1.50]$ & 0.017 & 0.051 & 0.01 & 0.031 & 0.025 \\
NAWK (times) & $15.00[5.50]$ & $0.089^{* *}$ & -0.012 & -0.053 & $0.065^{*}$ & $0.174^{* * *}$ \\
WASO (min) & $30.00[14.00]$ & $0.070^{*}$ & $-0.092^{* *}$ & -0.045 & 0.033 & $0.116^{* * *}$ \\
Sleep duration (min) & $409.00[57.50]$ & $0.222^{* * *}$ & $0.084^{* *}$ & $-0.092^{* *}$ & $0.163^{* * *}$ & $0.455^{* * *}$ \\
$\mathrm{~T}_{\text {mean }}$ (sec) & $256.09[51.17]$ & -0.026 & $0.062^{*}$ & 0.047 & -0.027 & -0.049 \\
$\mathrm{~T}_{\text {std }}$ (sec) & $304.78[57.81]$ & -0.025 & 0.029 & 0.046 & -0.041 & $-0.067 *$ \\
$\mathrm{~N}_{\mathrm{m}}$ (times) & $42.00[12.00]$ & $0.129^{* * *}$ & -0.042 & -0.048 & $0.080^{* *}$ & $0.237^{* * *}$ \\
$\mathrm{D}_{\mathrm{m}}$ (times/hr) & $6.21[1.35]$ & 0.026 & $-0.095^{* *}$ & -0.034 & 0.012 & 0.023 \\
Period (min) & $80.00[12.50]$ & $0.087 * *$ & -0.037 & -0.013 & $0.064 *$ & 0.048 \\
LIDS slope & $-7.99[5.95]$ & 0.043 & 0.015 & 0.052 & 0.026 & 0.054 \\
RoO & $7.68[1.93]$ & -0.032 & $-0.058^{*}$ & 0.026 & -0.051 & $-0.082^{* *}$ \\
$\mathrm{~N}_{\mathrm{c}}$ & $4.80[1.13]$ & $0.083^{* *}$ & $0.069^{*}$ & $-0.064 *$ & $0.068^{*}$ & $0.261 * * *$ \\
Circadian amplitude & $33.53[11.87]$ & -0.01 & -0.024 & -0.009 & -0.026 & -0.048 \\
Acrophase (hh:mm) & $16: 00[01: 41]$ & -0.003 & 0.005 & -0.008 & 0.014 & 0.009 \\
\hline
\end{tabular}

$* * * p<0.001, * * p<0.01, * p<0.05 S E$ sleep efficiency, $S O L$ sleep-onset latency, NAWK number of awakenings, WASO wake after sleep onset, $N_{\mathrm{m}}$ number of movements, $D_{\mathrm{m}}$ movement density, LIDS locomotor inactivity during sleep, $R o O$ range of oscillation in LIDS, $N_{\mathrm{c}}$ number of cycles in LIDS. These abbreviations and notations are applicable for the following tables

\begin{tabular}{|c|c|c|c|c|c|c|}
\hline SSQ/OSP & $\begin{array}{l}\text { Mean (SD) or } \\
\text { median [semi-iqr] }\end{array}$ & OSA-1 & OSA-2 & OSA-3 & OSA-4 & OSA-5 \\
\hline SE $(\%)$ & 94.84 [2.91] & 0.032 & $0.112 * *$ & 0.026 & 0.016 & 0.015 \\
\hline SOL (min) & $1.00[2.00]$ & 0.036 & 0.057 & 0.047 & 0.046 & -0.015 \\
\hline NAWK (times) & $11.00[5.00]$ & 0.038 & -0.036 & $-0.075^{*}$ & 0.066 & $0.173 * * *$ \\
\hline WASO (min) & $19.00[13.00]$ & 0.015 & $-0.094 * *$ & $-0.072 *$ & 0.03 & $0.116^{* *}$ \\
\hline Sleep duration (min) & $413.53(93.42)$ & $0.213 * * *$ & $-0.087 *$ & $-0.212 * * *$ & $0.195 * * *$ & $0.500 * * *$ \\
\hline $\mathrm{T}_{\text {mean }}(\mathrm{sec})$ & 299.86 [61.56] & -0.038 & $0.108 * *$ & 0.063 & -0.043 & $-0.074 *$ \\
\hline $\mathrm{T}_{\text {std }}(\mathrm{sec})$ & $348.85[65.88]$ & -0.023 & $0.102 * *$ & 0.042 & -0.042 & $-0.079 *$ \\
\hline $\mathrm{N}_{\mathrm{m}}$ (times) & $36.00[11.00]$ & $0.091^{*}$ & -0.053 & $-0.097 * *$ & $0.113 * *$ & $0.260 * * *$ \\
\hline $\mathrm{D}_{\mathrm{m}}($ times $/ \mathrm{hr})$ & $5.30[1.12]$ & -0.009 & $-0.122 * * *$ & -0.042 & 0.014 & 0.037 \\
\hline Period (min) & $80.00[12.50]$ & 0.046 & 0.057 & 0.007 & 0.052 & $0.084 *$ \\
\hline LIDS Slope & $-7.07[5.36]$ & -0.032 & -0.017 & -0.039 & 0.011 & $0.094 * *$ \\
\hline $\mathrm{RoO}$ & $6.72[1.63]$ & -0.005 & -0.052 & 0.013 & 0.008 & -0.025 \\
\hline $\mathrm{N}_{\mathrm{c}}$ & $5.19(1.48)$ & $0.088^{*}$ & 0.023 & $-0.088^{*}$ & $0.090 *$ & $0.237 * * *$ \\
\hline Circadian amplitude & $31.21[9.88]$ & -0.004 & -0.044 & -0.029 & -0.007 & -0.033 \\
\hline Acrophase (hh:mm) & $15: 33$ [01:30] & -0.048 & -0.037 & 0.024 & -0.013 & $-0.089 *$ \\
\hline
\end{tabular}

because of their similar age (Tables 2 and 3 ) and the worker analysis focused on generational difference within the males because of their widely distributed ages and because of the limited number of female worker subjects (Tables 4 and 5; for the middle generation group of workers $(35 \leq$ age $<45)$, see Table S-2 of Online Resource 2). The following tendencies were shared by the results for the students. (1) Sleep duration had non-negligible correlations with most of the OSA scores, especially OSA-1 (sleepiness at awakening), 4 (fatigue recovery), and 5 (subjective sleep duration). (2)
Parameters characterizing the total disturbances during sleep, such as NAWK, WASO, and $\mathrm{N}_{\mathrm{m}}$, exhibited similar profiles to those of sleep duration. (3) Associations with the parameters characterizing the occurrence density of disturbance such as SE and $\mathrm{D}_{\mathrm{m}}$ were confined to OSA-2 (sleep maintenance), as did the statistics of IMOIs, $\mathrm{T}_{\text {mean }}$ and $T_{\text {std }}$ in the female students. (4) Chronobiological and ultradian rhythm-related parameters such as amplitude and acrophase of circadian rhythm, period of ultradian rhythm, LIDS slope, and RoO were mostly uncorrelated with the 
Table 4 Spearman correlation (male workers, age $<35$ years) between daily quantitative sleep-related parameters and subjective sleep quality
Table 5 Spearman correlation (male workers, age $\geq 45$ years) between daily quantitative sleep-related parameters and subjective sleep quality

\begin{tabular}{llccccc}
\hline SSQ/OSP & $\begin{array}{l}\text { Mean (SD) or } \\
\text { median [semi-iqr] }\end{array}$ & OSA-1 & OSA-2 & OSA-3 & OSA-4 & OSA-5 \\
& & & & & \\
\hline SE (\%) & $91.58[3.74]$ & 0.082 & 0.018 & -0.02 & 0.055 & 0.063 \\
SOL (min) & $2.00[1.50]$ & -0.035 & -0.012 & 0.043 & 0.027 & 0.02 \\
NAWK (times) & $16.00[6.50]$ & 0.092 & 0.068 & -0.072 & $0.112^{*}$ & $0.221^{* * *}$ \\
WASO (min) & $31.00[18.13]$ & 0.009 & 0.02 & -0.044 & 0.041 & 0.081 \\
Sleep duration (min) & $390.6(94.8)$ & $0.284^{* * *}$ & $0.191^{* * *}$ & -0.076 & $0.283^{* * *}$ & $0.469^{* * *}$ \\
$\mathrm{~T}_{\text {mean }}$ (sec) & $266.58[55.36]$ & 0.023 & 0.031 & -0.013 & 0.016 & -0.084 \\
$\mathrm{~T}_{\text {std }}(\mathrm{sec})$ & $298.13[67.14]$ & -0.02 & -0.019 & 0.01 & -0.007 & -0.08 \\
$\mathrm{~N}_{\mathrm{m}}$ (times) & $37.00[11.50]$ & $0.128^{*}$ & 0.061 & -0.075 & $0.155^{* *}$ & $0.280^{* * *}$ \\
$\mathrm{D}_{\mathrm{m}}$ (times/hr) & $5.86[1.29]$ & -0.022 & -0.038 & -0.017 & 0.009 & 0.047 \\
Period (min) & $75.00[12.50]$ & 0.063 & 0.056 & -0.033 & 0.048 & 0.057 \\
LIDS slope & $-5.49[5.96]$ & 0.031 & -0.047 & 0.016 & 0.034 & 0.101 \\
RoO & $7.03[1.66]$ & 0.006 & -0.052 & 0.02 & 0.014 & -0.032 \\
$\mathrm{~N}_{\mathrm{c}}$ & $4.84[1.12]$ & $0.125^{*}$ & 0.075 & -0.058 & $0.130^{*}$ & $0.249 * * *$ \\
Circadian amplitude & $22.92[8.13]$ & 0.041 & -0.046 & -0.021 & 0.056 & 0.022 \\
Acrophase (hh:mm) & $15.00[1.50]$ & -0.025 & 0.029 & 0.022 & -0.024 & -0.035 \\
\hline
\end{tabular}

\begin{tabular}{llccccc}
\hline SSQ/OSP & $\begin{array}{l}\text { Mean (SD) or } \\
\text { median [semi-iqr] }\end{array}$ & OSA-1 & OSA-2 & OSA-3 & OSA-4 & OSA-5 \\
\hline SE (\%) & $90.91[5.43]$ & 0.01 & $0.106^{* * *}$ & 0.039 & 0.021 & 0.016 \\
SOL (min) & $3.00[1.50]$ & -0.007 & 0.07 & 0.003 & -0.019 & -0.04 \\
NAWK (times) & $14.00[7.00]$ & 0.072 & -0.028 & -0.066 & 0.055 & $0.167 * * *$ \\
WASO (min) & $31.00[21.50]$ & 0.029 & $-0.093^{*}$ & -0.056 & 0.034 & $0.099^{* *}$ \\
Sleep duration (min) & $377.47(83.32)$ & $0.173^{* * *}$ & 0.032 & $-0.086^{*}$ & $0.225^{* * *}$ & $0.406^{* * *}$ \\
$\mathrm{~T}_{\text {mean }}$ (sec) & $250.80[66.75]$ & 0.001 & $0.093^{*}$ & 0.049 & -0.018 & 0 \\
$\mathrm{~T}_{\text {std }}$ (sec) & $291.50[70.32]$ & -0.031 & $0.116^{* *}$ & $0.083^{*}$ & -0.008 & -0.052 \\
$\mathrm{~N}_{\mathrm{m}}$ (times) & $39.00[13.50]$ & 0.075 & -0.066 & -0.054 & $0.109 * *$ & $0.158^{* * *}$ \\
$\mathrm{D}_{\mathrm{m}}$ (times/hr) & $6.24[1.69]$ & 0.002 & $-0.102^{* *}$ & -0.034 & 0.021 & -0.018 \\
Period (min) & $75.00[12.50]$ & 0.028 & -0.045 & -0.016 & -0.005 & 0.014 \\
LIDS slope & $-4.76[5.98]$ & -0.059 & -0.062 & $-0.082^{*}$ & -0.005 & -0.015 \\
RoO & $7.07[1.68]$ & -0.037 & -0.055 & -0.011 & -0.064 & -0.045 \\
$\mathrm{~N}_{\mathrm{c}}$ & $4.71[1.05]$ & 0.057 & 0.021 & -0.054 & $0.130^{* * *}$ & $0.213^{* * *}$ \\
Circadian amplitude & $22.48[9.94]$ & -0.02 & -0.005 & -0.046 & 0.006 & $0.082^{*}$ \\
Acrophase (hh:mm) & $14: 20[01: 30]$ & 0.003 & -0.012 & -0.002 & -0.028 & -0.007 \\
\hline
\end{tabular}

OSA scores, although $\mathrm{N}_{\mathrm{c}}$ shared a profile with the total disturbance-related parameters. The correlation profile of female students differed from that of males in terms of nonnegligible significant correlations between sleep duration and OSA-3 (dreaming) and between statistics of IMOIs and OSA-2 (sleep maintenance). In addition, the total disturbance-related parameters showed slight correlation with OSA-4 only in female students, and with OSA-1 only in male students.

Compared with the students, the male workers showed non-negligible correlations of the parameters related to the total disturbances to OSA-4 as well as OSA-1. Slight but significant OSA-2-specific correlations of the disturbance density-related parameters, as a tendency shared by the students, were found only for the eldest worker group ( $>45$ years), as did the statistics of IMOIs. In addition, ultradian period had non-negligible correlations with OSA-1 and OSA-4 for the middle-aged group of workers.

Comparison between the students and youngest worker group revealed that sleep duration, the total disturbancesrelated parameters, and $\mathrm{N}_{\mathrm{c}}$ correlated with more OSA scores in the workers than in the students. In contrast, slight but significant OSA-2-specific correlations of the disturbance density-related parameters were found only in the students. 


\section{Classification of HSWPs}

Further analyses were performed to clarify how the subjective and quantitative aspects of sleep depend on HSWPs through clustering. The results of HSWP clustering are shown in Fig. 1, which shows day-to-day variations in sleepand wake-onset times superposed around the individual mean level, together with the typical HSWP, for each cluster. Together with the cluster-dependent statistical features of the parameters used for classification and the demographic a

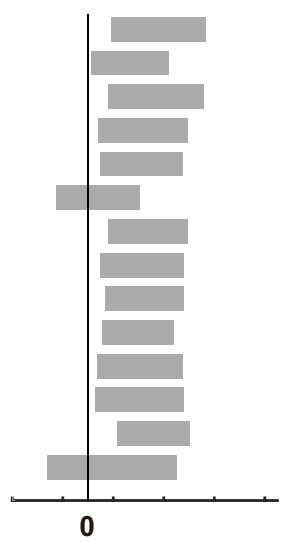

b
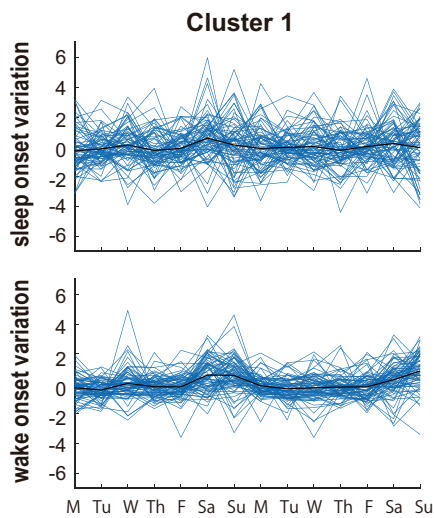

C

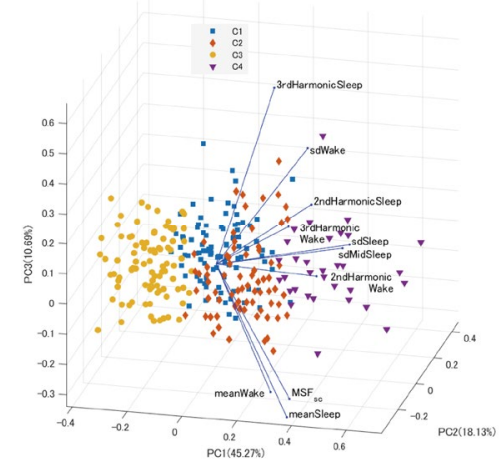

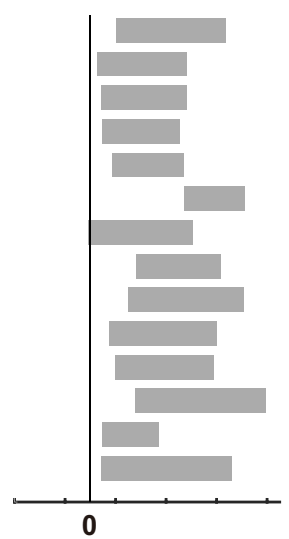

Cluster 2
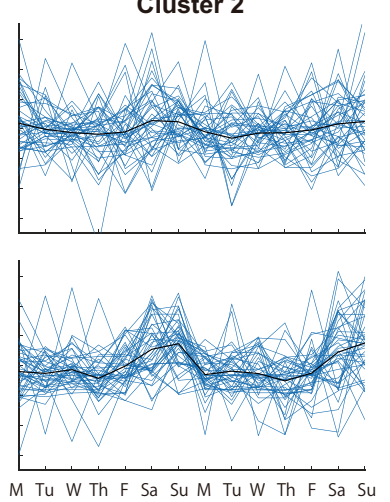

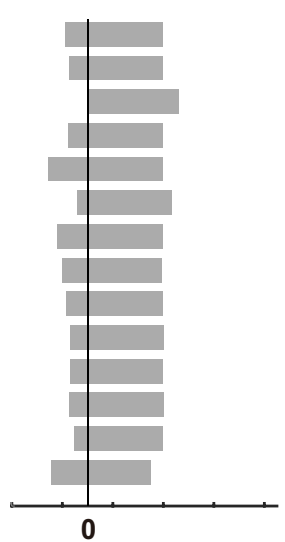

Cluster 3
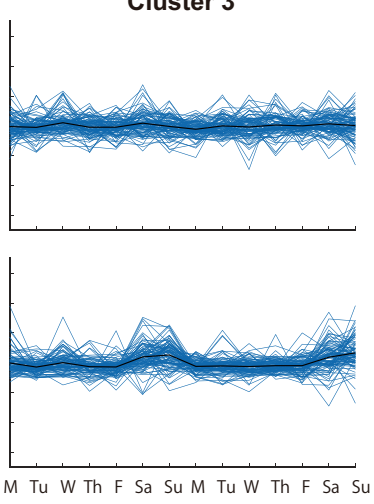

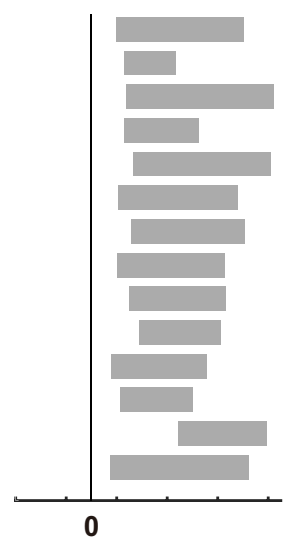

Cluster 4

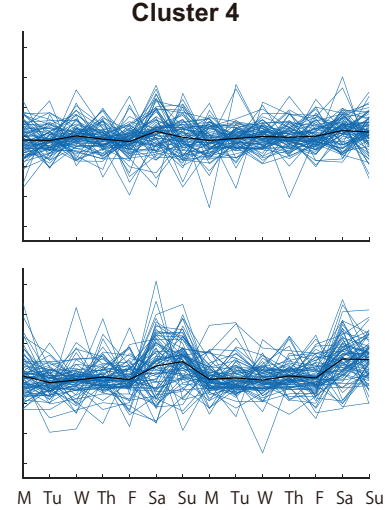

d

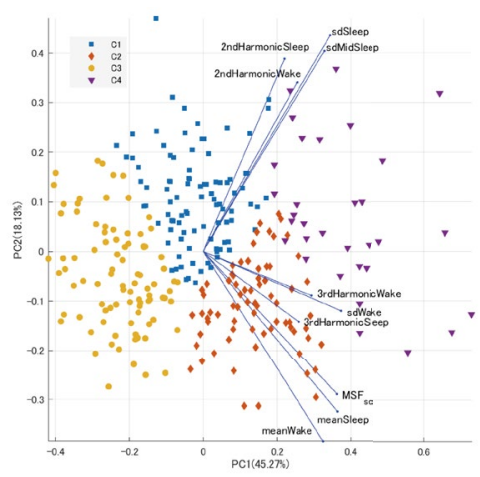

e

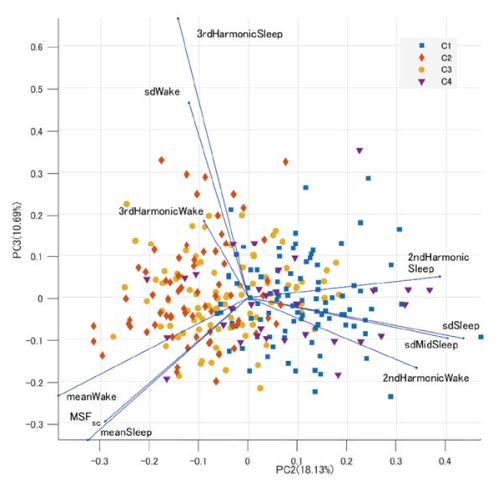

Fig. 1 a Typical sleep-wake patterns classified into the respective clusters of habitual sleep-wake patterns: from left to right, Clusters 1, 2, 3, and 4. b Day-to-day variations in sleep-onset (top) and wakeonset (bottom) times for the participants in each cluster superposed around the individual mean level (blue lines) and averaged traces (thick black line). c-e Distributions of sleep-wake patterns in the principal component (PC) coordinates from different views: c perspective view, d PC1-PC2 plane, and e PC2-PC3 plane. Colors correspond to the $\mathrm{C} 1-\mathrm{C} 4$ clusters of habitual sleep-wake patterns 
structure of clusters (Tables S-3 and S-4 of Online Resource 3 ), the HSWP clusters were characterized as follows:

(1) Cluster $1(\mathrm{C} 1, N=83)$ : The statistics for sleep- and wake-onset times, MS, and $\mathrm{MSF}_{\mathrm{sc}}$ showed that $\mathrm{C} 1$ shared the tendency of earlier habitual sleep with C3. Nevertheless, in contrast with C3, more severe SJL and greater irregularity of daily activity patterns in terms of SRI characterize this cluster. $\mathrm{C} 1$ or $\mathrm{C} 3$ included more workers than the other clusters, which was statistically suggested by Chi-square test of bias in demographic proportions across clusters.

(2) Cluster $2(\mathrm{C} 2, N=34)$ : $\mathrm{C} 2$ shared the tendency of later habitual sleep with C4. Extreme day-to-day and weekly variations in sleep- and wake-onset times distinguished $\mathrm{C} 2$ from $\mathrm{C} 4$, as did the irregularity of daily activity patterns. In $\mathrm{C} 2$ and $\mathrm{C} 4$, there were more students than workers. This bias of student proportions in $\mathrm{C} 2$ and $\mathrm{C} 4$ was suggested statistically.

(3) Cluster $3(\mathrm{C} 3, N=97)$ : This cluster was characterized by earlier habitual sleep with the most regular sleepand wake-onset times and the least SJL. Two-thirds of members of this cluster were workers.

(4) Cluster $4(\mathrm{C} 4, N=76)$ : This cluster was characterized by later habitual sleep, in which the day-to-day and weekly variations in sleep- and wake-onset times were less pronounced in comparison with $\mathrm{C} 2$. In addition, a slightly longer sleep duration distinguished it from the other clusters. Two-thirds of cluster members were students.

PCA conducted to represent the cluster features with a minimal dimension revealed the distribution of day-to-day variations in HSWPs in the third-order principal component (PC) coordinates (the total contribution ratio up to the third-order PC components was 74\%) together with the loading vectors, as shown in Fig. 1c-e. From mappings on the PC1-PC2 and PC1-PC3 planes, it was found that PC1 separated $\mathrm{C} 3$ and $\mathrm{C} 4$, and $\mathrm{PC} 2$ separated $\mathrm{C} 1$ and $\mathrm{C} 2$. From mapping on the PC2-PC 3 plane, $\mathrm{PC} 2$ separated $\mathrm{C} 1 / \mathrm{C} 4$ and $\mathrm{C} 2 / \mathrm{C} 3$. The PC1-PC3 vectors are listed in Table S-5 (Online Resource 3).

\section{Multiple dependency of subjective sleep quality and quantitative sleep-related parameters on demographic and HSWP characteristics}

The dependency of subjective sleep quality and quantitative sleep-related parameters on demographic characteristics shown in Table 1 was further analyzed by taking into consideration the HSWP clustering results and weekday/ weekend difference. Their multiple dependency on demographics (Gender and Job), Day (distinction of weekdays/ weekends), and HSWP clusters is shown up to 4-way interaction (Table 6). The significant effectors for the respective features are summarized graphically in Fig. 2. Subsequently, a post hoc analysis by Tukey-Kramer test was performed (Table S-6 of Online Resource 4). Based on these results, the sleep features significantly affected by the multi-way interactions are listed below, and their dependencies are explained, where the highest order of interactions is given in parenthesis with $* p<0.05, * * p<0.01$, or $* * * p<0.001$, and the abbreviations, $\mathrm{G}$ for Gender, J for Job, D for Day, and C for HSWP, are used.

OSA-4 and OSA-5 (D*): Subjective evaluation of fatigue recovery and sleep duration tended to be better on weekends in comparison with weekdays.

Sleep and wake onsets, and mid-sleep time $\left(\mathrm{C}: \mathrm{D}^{* * *}\right.$, $\left.\mathrm{J}: \mathrm{D}^{*}\right)$ : Sleep phase-related timings were found to exhibit the inter-cluster dependency similar to Table S-3, irrespective of weekdays or weekends. In addition, these timings tended to be significantly later on weekends than weekdays for almost all HSWP clusters. The students tended to wake up significantly later as compared with the workers, irrespective of weekdays or weekends.

Sleep duration (C:G:D*): Significant prolongation of sleep duration tended to take place on weekends for some combinations of cluster $(\mathrm{C} 2, \mathrm{C} 3, \mathrm{C} 4)$ and gender. For the females, weekend prolongation of sleep duration was pronounced for $\mathrm{C} 2$ in comparison with $\mathrm{C} 4$.

Circadian amplitude and acrophase (G:D*, C:D*): For the males, circadian amplitude tended to be larger on weekends than weekdays, and this weekend increase in circadian amplitude was shown to be more pronounced for the males. Weekday circadian acrophase tended to be earlier for $\mathrm{C} 3$ than $\mathrm{C} 2$.

SE, SOL, NAWK, and $\mathrm{D}_{\mathrm{m}}$ (J:D**, J:D*, C:G:D**, D*): The workers tended to experience lower weekend SE and longer weekend SOL, as compared with the students. $\mathrm{D}_{\mathrm{m}}$ tended to be higher on weekends. The male subjects belonging to $\mathrm{C} 3$ and $\mathrm{C} 4$ tended to experience more NAWK on weekends, as compared with the female workers.

Ultradian period and $\mathrm{N}_{\mathrm{c}}\left(\mathrm{C}: \mathrm{G}: \mathrm{D}^{*}, \mathrm{C}^{*}, \mathrm{D}^{* * *}\right)$ : Weekday ultradian period of the females belonging to $\mathrm{C} 2$ tended to be significantly longer than those to $\mathrm{C} 3 . \mathrm{N}_{\mathrm{c}}$ tended to be larger in $\mathrm{C} 3$ than $\mathrm{C} 4$, and on weekends than weekdays.

\section{Discussion}

The ability of demographic characteristics such as gender, job, and age to differentiate quantitative sleep-related features and subjective sleep quality and their associations has been shown previously [25-27]. In the present study, their demographics dependency was further analyzed. To quantitatively characterize activity during sleep, IMOIs and 


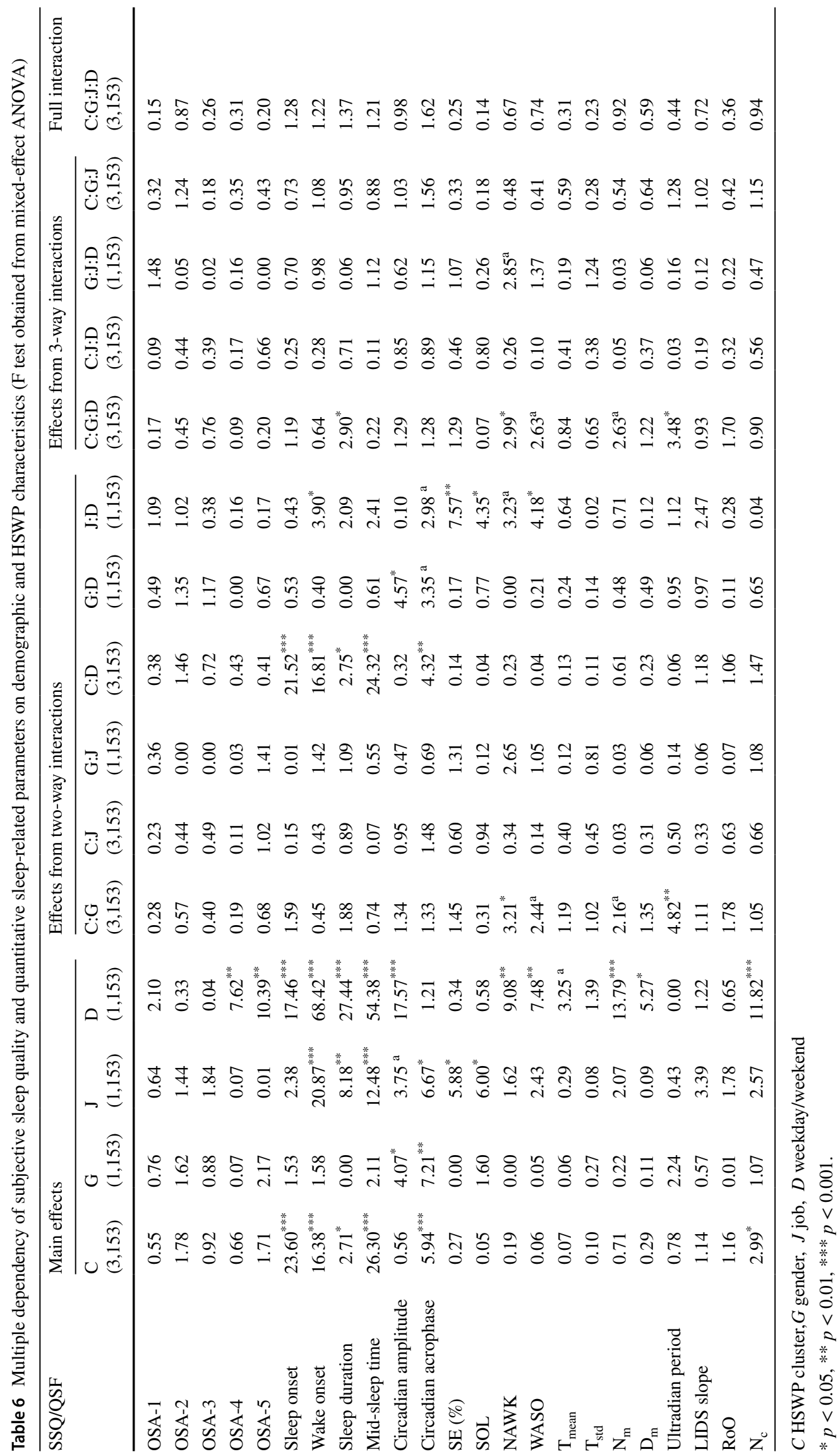




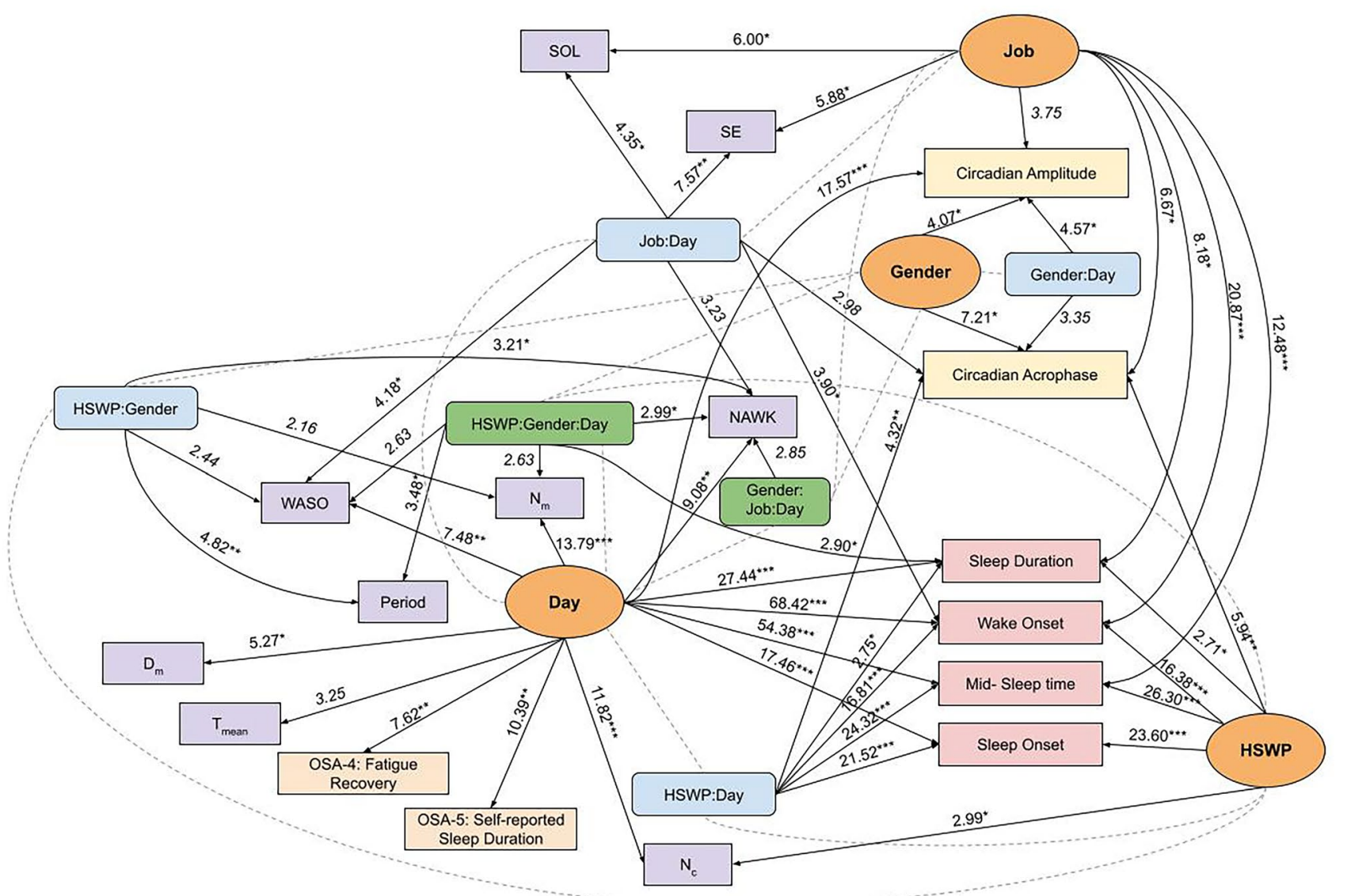

Fig. 2 Graphical representation of dependency of quantitative sleeprelated parameters and subjective sleep quality on the demographic characteristics, distinction of weekdays/weekends, HSWP, and their multi-way interactions. Orange ovals: factors to be tested, pink squares: subjective sleep quality and quantitative sleep-related parameters; blue squares: 2-way interactions; green squares: 3-way inter-

ultradian inactivity rhythms were estimated, in addition to the conventional parameters produced by the accelerometer algorithm [19, 21]. In our analysis, longer IMOIs responsible for body sway or posture change other than jitter-like events [28] were extracted. Ultradian inactivity rhythms were characterized based on a similar range of parameters reported in a previous study [21]. In part, our results shared a previously reported tendency for students to take a later, longer, and more irregular sleep compared with workers, irrespective of gender $[10,13,27]$. Despite these features, student's sleep was found to be less disturbed than that of the workers. This paradoxical finding might be due to the worker's obligatory regularity of lifestyle, which is suggested by the pronounced SJL for the workers (Table 1), although there is a possibility of confoundment by age. Regarding the gender difference, the male students tended to take a later and more disturbed sleep compared with the female students. There were more dense disturbances in the sleep of male workers than female workers. Although less distinctive, some scores of subjective actions. A significant level of each effect represented by an arrow is denoted by $F$-values with $* p<0.05$, ** $p<0.01$, *** $p<0.001$. The numbers with no asterisk indicate a tendency only, with $p<0.1$. Dashed lines indicate factors that combine to form multi-way interactions

sleep quality tended to be lower in the female subjects than in the males. Taken together, female subjects have tended to self-evaluate their sleep quality as worse than the quantitative features of less disturbed sleep in comparison with male subjects [21, 27, 29, 30]. Worker's age dependency of sleep features revealed that the middle-aged group tended to have less disturbed sleep in a subjective as well as objective sense, but less satisfactory sleep in comparison with the other generation groups. In addition, the eldest generation group in other studies has also exhibited earlier HSWP and a flatter ultradian slope compared with younger groups [20, 21].

Analysis of associations between quantitative sleeprelated parameters and subjective sleep quality for each demographic group showed non-negligible correlations of student's sleep duration with OSA scores except OSA-2 (sleep maintenance), which was also the case for parameters characterizing the total disturbances during sleep. This coincidence could be understood particularly when the occurrence density of disturbances shows not so much variation 
through an individual's sleep. In contrast, there was an association of OSA-2 with parameters characterizing the occurrence density of disturbance. The present study is the first to reveal a categorical profile of associations between the subjective and quantitative features of sleep. Gender difference analysis has added the female-specific characteristics of non-negligible significant OSA-3 (dreaming)-related correlations to some quantitative feature parameters of sleep [31]. Compared with the students, for the male workers, non-negligible correlations of the parameters related to the total disturbances were found additionally for OSA-4 (fatigue recovery), in all generation groups. The eldest worker group ( $\geq 45$ years) exhibited a slight but significant correlation of the disturbance density-related parameters with OSA-2, which was also shared by the students. That this tendency was shared by all generations of workers might suggest their awareness of fatigue recovery. In the job comparison between the male students and workers aged $<35$ years, sleep duration was non-negligibly correlated also with OSA-2 for the workers in contrast to the students whose disturbance density-related parameters were found to be correlated only with OSA-2. The students might be more sensitive than the workers to disturbances partly, because the students' sleep is usually less disturbed.

The above analysis showed the fundamental demographics dependency of qualitative and quantitative features of sleep, and their associations. Nevertheless, the demographics dependency was mostly confined to the quantitative features, and the moderate association profiles were not sufficient to fully explain the behavior of sleep quality. To investigate another aspect of sleep, HSWPs were then characterized by clustering. Our classification of HSWPs revealed four clusters, $\mathrm{C} 1-\mathrm{C} 4$, which enabled differentiation of the relationship between quantitative sleep-related features and subjective sleep quality. Among the various indices of the static and dynamical aspects of HSWPs [2, $3,8]$, chronotype was used directly for our classification; the second harmonics of the sleep- and wake-onset times included information on SJL, and SRI was in part shared by SDs and harmonics of the sleep- and wake-onset times. In this sense, our classification was conducted through a composite version of these conventional measures. Testing of bias in demographic proportions across clusters revealed significant biases in job and age group but not in gender (Table S-4 in Online Resource 3). C1 and C3 shared a similar tendency of earlier habitual sleep. In contrast, C2 and C4 shared a similar tendency of later habitual sleep. This cluster dependency might be at least partly due to the demographic structure of clusters that more workers (elderly subjects) in the former clusters and more students (younger subjects) in the latter clusters were included, respectively, considering the job- and age-dependent sleep features known previously $[20,32]$. In other words, patterns of day-to-day variations in wake- and sleep onsets and SJL, among others, are thought to further differentiate detailed classification. The PCA further provided a reduced dimensional representation of the clusters formed by the composite measures.

By additionally including the results of HSWP classification, subjective sleep quality and quantitative features of sleep were shown to depend in multiple ways on demographics (Gender and Job), HSWP clustering results, and distinction of weekdays/weekends (Day). Further details of dependencies were analyzed by the post hoc test. Sleep phase-related timings were found to exhibit the inter-cluster dependency similar to Table S-3, irrespective of weekdays or weekends. Common habit of SJL was well recognized from the tendency that most of these timings were more delayed on weekends in comparison with weekdays [22,33] In addition, the workers tended to wake up earlier than the students [32]. Chronobiological features of daily activity revealed the gender-specific tendency that the male's circadian amplitude was larger on weekends than weekdays $[10,20]$. Circadian acrophase characterized $\mathrm{C} 2$ as the cluster with significantly later HSWP than the others. Sleep duration was found to be significantly prolonged on weekends within some HSWP clusters in a gender-specific manner. This could be understood at least partly from the common lifestyle causing SJL [22, 33]. Increasing tendency of $\mathrm{N}_{\mathrm{c}}$ on weekends might be associated with the prolongation of sleep duration because of no significant effect of day on the ultradian period. Disturbances were more pronounced on the weekend sleep of workers than the students. Especially for relatively regular HSWP clusters, the male's weekend sleep tended to be more disturbed than the weekday sleep. Such paradoxical tendency of disturbed weekend sleep for relatively regular HSWPs needs further investigation to be clarified. On the other hand, subjective sleep quality in terms of OSA-4 and OSA-5 tended to be better on weekends than weekdays. This tendency suggests multifaceted relationship between quantitative features of sleep and subjective sleep quality.

This study has some limitations. The actigraphy data analyzed here were collected from university students and information technology workers. However, the demographic balance of subjects was not well organized. The ages of female workers were unevenly distributed, which made age-related comparison within them difficult. Age distribution of female workers needs to be further diversified. In addition, the subjective sleep quality and the quantitative sleep features were not fully explained by the demographics and HSWP. Such a disagreement has commonly been recognized, although the sleep quality questionnaires used previously were different from those in the present study [6, 34]. More elaborate analysis and additional observations might be necessary to improve the predictability of sleep quality, and will be a topic of future study. There seems to be inconsistency in usage of statistics that the non-parametric test was used in 
Table 1, and the multi-way ANOVA in Table 6. However, the multi-way ANOVA has been empirically known to be robust against non-normality at least for lower order interactions [24, 25]. In addition, we confirmed that the residuals of the multiple dependency analysis did not so seriously deviate from the normality by a quantile-quantile plot. Highly accurate and elaborate analysis taking non-normality into account will be a future subject [35].

\section{Conclusions}

Subjective sleep quality was evaluated in light of various aspects-based OSA scores, each of which was associated in a particular way with quantitative sleep-related parameters. The associations were differentiated according to HSWP clusters. Further quantitative dissection of subjective sleep quality, considering multifaceted factors, such as gender, age, and occupation, could improve the validity of the present results, and enable selection of an HSWP fitted to an individual's working style and preferences.

Supplementary Information The online version contains supplementary material available at https://doi.org/10.1007/s41105-021-00326-9.

Acknowledgements This work was supported by a grant from the JST Center of Innovation Program (COI) (No. JPMJCE1303).

\section{Declarations}

Conflict of interest The authors declare that they have no conflict of interest.

Ethical committee permission All procedures involving human participants were performed in accordance with the ethical standards of the Ethical Committee of the Graduate School of Information Sciences, Tohoku University, and with the 1964 Helsinki Declaration and its later amendments.

Informed consent Informed consent was obtained from all individuals who participated in this study.

Open Access This article is licensed under a Creative Commons Attribution 4.0 International License, which permits use, sharing, adaptation, distribution and reproduction in any medium or format, as long as you give appropriate credit to the original author(s) and the source, provide a link to the Creative Commons licence, and indicate if changes were made. The images or other third party material in this article are included in the article's Creative Commons licence, unless indicated otherwise in a credit line to the material. If material is not included in the article's Creative Commons licence and your intended use is not permitted by statutory regulation or exceeds the permitted use, you will need to obtain permission directly from the copyright holder. To view a copy of this licence, visit http://creativecommons.org/licenses/by/4.0/.

\section{References}

1. Minlek JD, Banks S, Htaik O, Moreta MC, Jones CW, McGlinchey EL, et al. Sleep deprivation and stressors: Evidence for elevated negative affect in response to mild stressors when sleep deprived. Emotion. 2012;12(5):1015-20.

2. Roenneberg T, Allebrandt KV, Merrow M. Social jetlag and obesity. Curr Biol. 2012;22(10):939-43.

3. Lunsford-Avery JR, Engelland MM, Navar AM, Kollins SH. Validation of the sleep regularity index in older adults and associations with cardiometabolic risk. Sci Rep. 2018;8:14158.

4. Nishino S. Stanford Method for Ultimate Sound Sleep (in Japanese). Tokyo: Sunmark Publishing Inc; 2017.

5. Sasaki JE, John D, Freedson PS. Validation and comparison of ActiGraph activity monitors. J Sci Med Sport. 2011;14:411-6.

6. Sadeh A. The role and validity of actigraphy in sleep medicine: An update. Sleep Med Rev. 2011;15:259-67.

7. Watson NF, Badr MS, Belenky G, Bliwise DL, Buxton OM, Buysse D, et al. Recommended amount of sleep for a healthy adult: A joint consensus statement of the american academy of sleep medicine and sleep research society. Sleep. 2015;38(6):843-4.

8. Roenneberg T, Wirz-Justice A, Merrow M. Life between clocks: Daily temporal patterns of human chronotypes. J Biol Rhythms. 2003;18:80-90.

9. Paine SJ, Gander PH. Differences in circadian phase and weekday/weekend sleep patterns in a sample of middle-aged morning types and evening types. Chronobiol Int. 2016;33(8):1009-17.

10. Vitale JA, Roveda E, Montaruli A, Galasso L, Weydahl A, Caumo A, et al. Chronotype influences activity circadian rhythm and sleep: Differences in sleep quality between weekdays and weekend. Chronobiol Int. 2015;32(3):405-15.

11. Wittmann M, Dinich J, Merrow M, Roenneberg T. Social jetlag: misalignment of biological and social time. Chronobiol Int. 2006;23(1-2):497-509.

12. Phillips AJK, Clerx WM, O’Brien CS, Sano A, Barger LK, Picard RW, et al. Irregular sleep/wake patterns are associated with poorer academic performance and delayed circadian and sleep/wake timing. Sci Rep. 2017;7(1):1-13.

13. Soehner AM, Kennedy KS, Monk TH. Circadian preference and sleep-wake regularity: Associations with self-report sleep parameters in daytime-working adults. Chronobiol Int. 2011;28(9):802-9.

14. Nakao M, Karashima A, Katayama N. Mathematical models of regulatory mechanisms of sleep-wake rhythms. Cell Mol Life Sci. 2007;64:1236-43.

15. Bei B, Wiley JF, Trinder J, Manber R. Beyond the mean: A systematic review on the correlates of daily intraindividual variability of sleep/wake patterns. Sleep Med Rev. 2016;28:108-24.

16. Amano R, Karashima A, Motoike I, Katayama N, Kinoshita K. Consistency index of daily activity pattern and its correlations with subjective ratings of QOL. Sleep Biol Rhythms. 2020;18:297-304.

17. Yamamoto Y, Tanaka H, Takase M, Yamazaki K, Azumi K, Shirakawa S. Standardization of revised version of OSA sleep inventory for middle age and aged. Brain Sci Mental Disorders. 1999;10:401-9.

18. Cole RJ, Kripke DF, Gruen W, Mullaney DJ, Gillin JC. Automatic sleep/wake identification from wrist activity. Sleep. 1992;15(5):461-9.

19. ActiGraph wGT3X-BT. [Online]. [cited 2018 April 10. Available from: https://actigraphcorp.com/actigraph-wgt3x-bt/.

20. Roenneberg T, Kuehnle T, Pramstaller PP, Ricken J, Havel M, Guth A, et al. A marker for the end of adolescence. Curr Biol. 2004;14(24):R1038-9. 
21. Winnebeck EC, Fischer D, Leise T, Roenneberg T. Dynamics and ultradian structure of human sleep in real life. Curr Biol. 2018;28(1):49-59.

22. Pilz LK, Keller LK, Lensen D, Roenneberg T. Time to rethink sleep quality: PSQI scores reflect sleep quality on workdays. Sleep. 2018;41(5):zsy029.

23. Han J, Kamber M, Pei J. Data Mining. Middlesex: Morgan Kaufmann; 2012.

24. Harwell MR, Rubinstein EN, Hayes WS, Olds CC. Summarizing Monte Carlo results in methodological research: The one- and two-factor fixed effects ANOVA cases. J Educational Statistics. 1992;17(4):315-39.

25. Park YM, Matsumoto K, Shinkoda H, Nagashima H, Kang MJ, Seo YJ. Age and gender difference in habitual sleep-wake rhythm. Psychiatry Clin Neurosci. 2001;55(3):201-2.

26. Lehnkering H, Siegmund R. Influence of chronotype, season, and sex of subject on sleep behavior of young adults. Chronobiol Int. 2007;24(5):875-88.

27. Park YM, Seo YJ, Matsumoto K, Shinkoda H, Park KP. Sleep in relation to age, sex, and chronotype in Japanese workers. Percept Mot Skills. 1998;87(1):199-215.

28. Yoneyama M, Okuma Y, Utsumi H, Terashi H, Mitoma H. Human turnover dynamics during sleep: Statistical behavior and its modeling. Phys Rev E. 2014;89(3):032721.

29. Goel N, Kim H, Lao RP. Gender differences in polysomnographic sleep in yound healthy sleepers. Chronobiol Int. 2005;22(5):905-15.
30. Tsai LL, Li SP. Sleep patterns in college students: Gender and grade differences. J Psychosom Res. 2004;56(2):231-7.

31. Selvi Y, Aydin A, Gulec M, Boysan M, Besiroglu L, Ozdemir PG, et al. Comparison of dream anxiety and subjective sleep qualitybetween chronotypes. Sleep Biol Rhythms. 2012;10:14-22.

32. Park YM, Seo YJ, Matsumoto K, Shinoda H, Park KP. Scores on morningness-eveningness and sleep habits of Korean students, Japanese students, and Japanese workers. Percept Mot Skills. 1997;85(1):143-54

33. Roepke SE, Duffy JF. Differential impact of chronotype on weekday and weekend sleep timing and duration. Nature Sci Sleep. 2010;2:213-20.

34. Goelema MS, Regis M, Haakma R, van den Heuvel ER, Markopoulos $\mathrm{P}$, Overeem $\mathrm{S}$. Determinants of perceived sleep quality in normal sleepers. Behav Sleep Med. 2019;17(4):388-97.

35. Schmider E, Ziegler M, Danay E, Beyer L, Bühner M. Is it really robust? Reinvestigating the robustness of ANOVA against violations of the normal distribution assumption. Methodology. 2010;6:147-51.

Publisher's Note Springer Nature remains neutral with regard to jurisdictional claims in published maps and institutional affiliations. 